\title{
Classification of Toxic Cyanobacterial Blooms by Fourier-Transform Infrared Technology (FTIR)
}

\author{
Gabriel Kenne, Deon van der Merwe \\ Kansas State University, Manhattan, USA \\ Email: gkenne@email.sc.edu
}

Received July 25, 2013; revised August 26, 2013; accepted September 3, 2013

Copyright (C) 2013 Gabriel Kenne, Deon van der Merwe. This is an open access article distributed under the Creative Commons Attribution License, which permits unrestricted use, distribution, and reproduction in any medium, provided the original work is properly cited.

\begin{abstract}
Cyanobacteria are gram-negative photosynthetic bacteria capable of producing toxins responsible for morbidity and mortality in humans and domestic animals. They are capable of forming concentrated blooms, referred to as harmful algal blooms (HABs). Characterization of HABs is necessary to reduce risks from human and animal exposures to toxins. Current methods used to classify cyanobacteria and cyanotoxins have limitations related to time, analyst skills, and cost. Fourier-Transform Infrared Spectroscopy (FTIR) is a potential tool for rapid, robust cyanobacterial classification that is not limited by these factors. To examine the practicality of this method, library screening with default software algorithms was performed on HAB samples, followed by principle component cluster analyses and dendrogram analysis of samples meeting minimum quality requirements. Two tested spectrometers and software packages were successful at distinguishing cyanobacteria from green algae. Principle component cluster analysis and dendrogram analysis also resulted in clear differentiation between cyanobacteria and green algae. While these methods cannot be used independently to fully characterize HABs, they show the potential and practicality of FTIR as a screening tool.
\end{abstract}

Keywords: Cyanobacteria; Harmful Algal Bloom; Fourier-Transform Infrared Spectroscopy

\section{Introduction}

Cyanobacteria are gram-negative photosynthetic prokaryotes. At least 2,654 species have been classified worldwide [1]. They are also known as blue-green algae, and are normally found in water systems across the world. Several genera are able to produce toxins, referred to as cyanotoxins, which pose a health risk to animals and humans $[2,3]$. In fresh water bodies, favorable growth conditions can allow cyanobacteria to form large blooms, and due to the buoyancy of some species they are often seen as bright green or blue-green scums on the water surface. Blooms may contain one dominant species, or several species and genera of cyanobacteria, with both situations capable of producing more than one type of cyanotoxin [4]. Not all blooms are toxic, however, and the environmental parameters that influence the internal dynamics of these cyanobacterial communities and their toxin production remain poorly understood $[5,6]$.

Cyanotoxins can be classified based on their chemical structure and their toxic effects, including the cyclic peptides such as hepatotoxic microcystins, alkaloid neurotoxins, lipopolysaccharides, and others [2,3]. Cyanotoxins are of concern to human health if they are present in consumed water. This ingestion hazard has led to the World Health Organization setting a guideline of $1 \mu \mathrm{g} / \mathrm{L}$ of microcystin in drinking water and cell count guidelines for recreational use waters warning of moderate health risks when greater than 100,000 cells $/ \mathrm{mL}[3,4]$. Cyanotoxins can also have a severe effect on domestic animals and livestock that drink from lakes and ponds. Livestock and other animals often do not avoid drinking from water containing cyanobacterial blooms, leading to numerous reports of livestock and companion animal morbidity and mortality in the literature [7,8]. Toxic cyanobacterial blooms also create a public health risk to recreational users of public waters. While accidental ingestion does occur, the primary exposure seen in humans is dermal and mucous membrane contact. Gamboro et al. describe that cyanobacterial cells and toxins can be aerosolized by boating or whitecaps and may pose an indirect exposure threat to recreational lake users $[2,9]$. Since cyanobacterial toxins have the potential to cause severe illness and even death to animals and humans, efficient characterization of potentially toxic cyanobacterial blooms is needed. 
Many methods are currently used for the detection of cyanobacteria and their toxins. Microscopy can be a relatively inexpensive method to identify cyanobacterial cells from water samples, and remains the standard diagnostic method. However, the need for skilled microscopists is a limiting factor. Some microscopy methods, such as electron microscopy, are impractical for large sample numbers that require more efficient detection methods [10]. Two detection methods generally employed for toxin identification and quantification are ELISA and liquid chromatography/mass spectrometry (LC/MS) [2]. ELISA allows for the detection of cyanotoxins for which antibodies have been synthesized and tested [11]. Regarding the use of liquid chromatography, Mackey et al. have developed a method for calculating algal class abundances from measurements of chlorophyll and carotenoid pigments by HPLC [12]. LC/MS pigment methods are limited, however, since some pigments are unambiguous for certain classes, but many are present in several classes [10]. More advanced mass spectroscopy methods have been described for cyanotoxin detection that overcome some of the drawbacks of conventional LC/MS methods, i.e. response to individual congeners, sensitivity between analyses, and linearity of the standard curves by the use of smaller column media and tandem mass spectrometry [4].

More recently, methods utilizing polymerase chain reaction techniques (PCR, qPCR) have been developed to detect the presence of microcystin-synthesizing genes, such as mcy, that are present in cyanobacterial species capable of producing the microcystin toxin [5,6]. Identification of these genes is not always indicative of toxicity though, because qPCR only gives an indication of the number of organisms potentially capable of synthesizing microcystin $[5,6]$. Protein phosphatase inhibition assays have also been developed for the detection of microcystins [13]. While many of these methods are successful at identifying cyanobacteria and their toxins, they may not always be practical because they require highly trained staff, expensive equipment and reagents, or time-consuming sample preparations.

A method that has proven useful for efficient and inexpensive bacterial characterization is Fourier-Transform Infrared Spectroscopy (FTIR). Sacksteder et al. describe FTIR as a form of vibrational spectroscopy whose spectrum reflects both molecular structure and molecular environment [14]. FTIR has been used to investigate physiological effects of nitrogen deprivation on diatoms, to study nutrient-related changes in various cyanobacteria and diatoms, as well as in the discrimination of cyanobacterial strains [15-18]. FTIR spectroscopy of phytoplankton results in complex absorption spectra that provide qualitative and quantitative data on freshwater systems [19]. The infrared spectra of cells produced by FTIR techniques give distinct, highly reproducible patterns that are unique for different cells, allowing for taxonomic differentiation to be performed [17]. These infrared spectra are very complex, resulting from contributions of all cellular macromolecules, with the exact frequency of each vibration depending on the strength of the bonds involved, the mass of the component atoms, and the chemical environment of the molecular groups $[15,16]$. Here, FTIR is applied as a rapid, reliable, and cost-effective method to classify and differentiate potentially harmful cyanobacteria from green algae and other phytoplankton in dried algal scum samples.

\section{Methods}

\subsection{Sample Collection and Preparation}

Forty-six algal scum samples were derived from water samples submitted to the Kansas State Veterinary Diagnostic Laboratory (KSVDL) from August of 2011 through September of 2012. Water samples were from various lakes and ponds throughout the Midwest that were suspected of containing toxic cyanobacteria. Twenty additional samples were collected from water bodies near Manhattan, KS, and included other types of green material similar in appearance to cyanobacteria when placed on filter paper, such as latex paint and pureed grass or leaves. All water samples were examined under a light microscope to determine the presence of cyanobacteria. Samples diagnosed as positive for the presence of cyanobacteria, as well as green algae and select negative samples were then used for the FTIR analysis on two FTIR instruments.

A small amount of the sample was filtered through Watman \#4 filter paper to separate solid material from the water in the sample. Alternatively, for samples that contained a visible scum, scum material was spread onto the filter paper using a spatula. The filter papers were covered in algal cells until no paper was visible over an area of at least $1 \mathrm{~cm}^{2}$, and then left to air dry overnight (Figure 1). The data generated by the two instruments from the 46 KSVDL samples were labeled OMNIC, and OPUS, respectively. Eight cyanobacterial and green algae samples from the additional sample set collected near Manhattanwere later added to the OMNIC data set, resulting in a total of 54 OMNIC samples and 46 OPUS samples.

\subsection{FTIR Spectroscopy}

The attenuated total reflectance IR spectrum of each sample was obtained using both a Bruker Alpha-P with a diamond ATR module (Bruker Optics Inc., Billerica, MA), and a Nicolet 6700 (Thermo Fischer Scientific Inc., Waltham, MA) FTIR spectrometer fitted with a GladiATR diamond ATR module (Pike Technologies, Madison, WI). Absorbance spectra were measured over the wavenumber range of $4000-400 \mathrm{~cm}^{-1}$ with a spectral reso- 


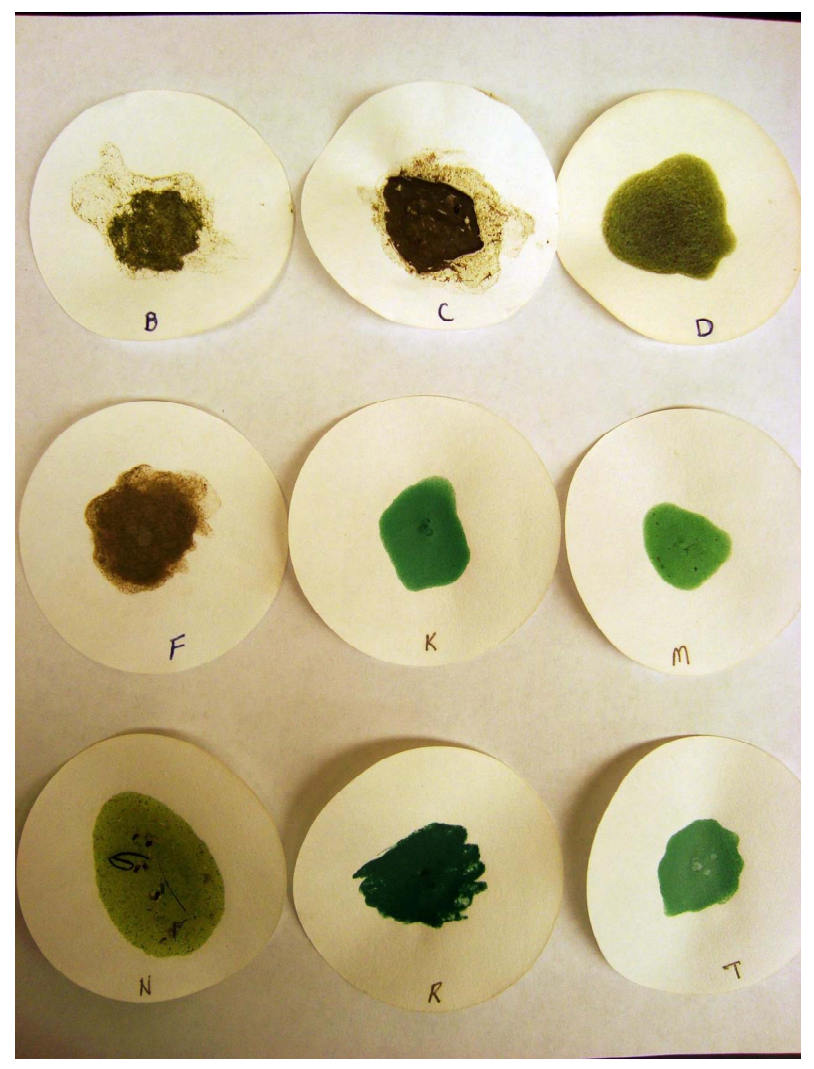

Figure 1. Select samples prepared for FTIR spectroscopy.

lution of $4 \mathrm{~cm}^{-1}$ on both spectrometers. The absorbance was measured over 24 and 32 scans on the Bruker and Nicolet, respectively.

The aperture crystal surface was cleaned with isopropanol and dried, followed by a background scan prior to the scanning of sample sets. All of the 46 initial samples for the primary analysis from both data sets were analyzed in triplicate on both the Nicolet and Bruker instruments. Twelve samples that were initially analyzed in August of 2011, using only the Nicolet 6700, were analyzed again during 2012 to determine if the samples degraded over time. The 20 samples used for the additional analysis were each scanned once per sample, using only the Nicolet 6700 .

\subsection{Software Default Algorithm Screening}

Samples containing a single genus of cyanobacteria or green algae, confirmed by microscopy, were added to spectral libraries present in both Nicolet's OMNIC (Thermo Fischer Scientific Inc., Waltham, MA) and Bruker's OPUS (Bruker Optics Inc., Billerica, MA) software programs. Each sample was screened against these libraries and a list of the closest related library spectra was generated based on the default software algorithm settings from each software program. Three negative/ debris samples, that were neither green algae nor cyano- bacteria, were removed from the data set and resulted in a total sample size of 43 for both the OMNIC and OPUS data sets, 31 of which contained only one genus of cyanobacteria. The OMNIC software default algorithm performs a spectral correlation of wavenumbers 2600 400 , while the OPUS software performs correlation on the entire spectrum. The top two spectra generated and their calculated match values for each sample were then recorded and used in the analysis.

The twelve repeated samples in the OMNIC data set were used in a separate algorithm screening that was compared to the results of the same samples scanned in 2011. The final group of 20 samples was screened in the same manner, but to test the reproducibility of the method they were scanned and screened by a laboratory member unassociated with the project.

\subsection{Principle Component Analysis}

Twelve peaks between 3280 and $1150 \mathrm{~cm}^{-1}$ were used for statistical analysis (Figure 2). Raw peak absorbance values were recorded for all clearly defined peaks within the samples. Peak height values were normalized to the Amide I band and analyzed using MATLAB ${ }^{\circledR}$ 7.7.0.471 (The Mathworks Inc., Natick, MA). Samples containing only a single genus were separated from mixed cyanobacterial samples for principle component and cluster analyses. Poor quality sample spectra were eliminated prior to these analyses based the raw absorbance intensity of the Amide I band. OMNIC spectra with an Amide I peak absorbance of $<0.10$ and OPUS spectra $<0.06$ were eliminated from the data sets, resulting in 40 single-genus OMNIC and 31 single-genus OPUS samples to be analyzed.

Principle component cluster analysis was performed on MATLAB $^{\circledR}$ 7.7.0.471 software using all FTIR spectra (including all triplicates) for each data set. After application of the described exclusion criteria, the OMNIC data set used a total of 107 spectra including the twelve 2012 repeat spectra, and the OPUS data set used a total of 91. Because only clearly defined peaks were recorded from the FTIR spectra, there were some absent peak values within the data sets for wavenumbers that were masked by adjacent peaks. To prevent the software from using a value of zero for these peaks, absent peak values were replaced with the average value of that peak for each genus. Zero values were allowed only when no masking peak was present.

\subsection{Dendrograms}

MATLAB $^{\circledR}$ 7.7.0.471 was also used to create dendrograms using average Euclidean distance hierarchical clustering for each set of data to express the dissimilarities between the spectra. Average spectra were generated for each genus in both data sets to create dendrograms 


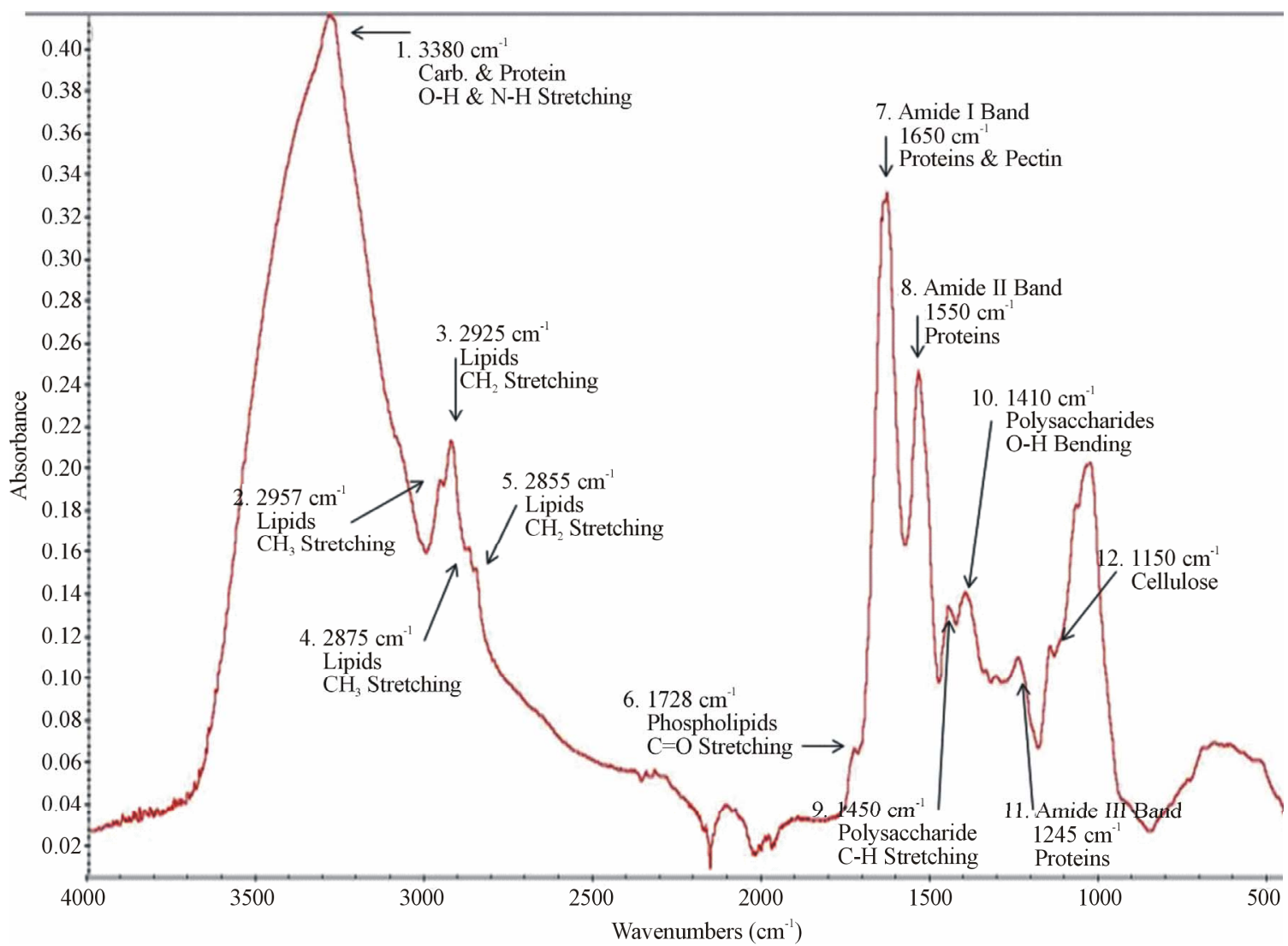

Common FTIR wavenumbers used for examining plants, bacteria, and cyanobacteria. Each peak represents a specific molecular movement and includes the parts of functional groups from which they are derived [15-17,20,21].

Figure 2. Absorbance peaks used for spectral analysis.

expressing the differences between the five cyanobacterial genera and green algae. These average spectra, however, do not express any variation seen within the samples of each genus. To observe this variation, dendrograms were also created for the each whole sample set using a single spectrum from each sample.

\section{Results}

\subsection{Cyanobacterial Discrimination from Green Algae}

Both software programs were successful at differentiating between cyanobacteria and green algae through the standard library screening algorithms. To be considered a success, both of the top two matches for all three triplicate spectra for a sample had to be correctly identified as cyanobacteria by the software. The green algae samples required the same match requirements for all three triplicate spectra, but were matched to the green algae control. The OPUS software correctly classified 36 of the 43 total samples $(83.72 \%)$ and 29 of the 31 single genus samples $(93.55 \%)$. The OMNIC software provided very similar results with 35 of 43 total samples $(81.40 \%)$ and 28 of 31 single genus samples $(90.32 \%)$ correctly classified. Per- cent agreement between the two spectrometers and their software packages was $89.13 \%$. The twelve OMNIC samples that were re-scanned after one year had a $100 \%$ match with the results of the initially scanned samples. The same 10 samples in this data set were correctly classified in both 2011 and 2012.

Of the 20 additional samples analyzed on the Nicolet 6700, all 10 cyanobacterial samples, and 3 of the 5 green algae samples, were correctly classified. The remaining 2 green algae samples contained high levels of debris and mud, and were not classified as either cyanobacteria or green algae by the library search algorithms. The remaining 5 samples consisted of green latex paint, a high density protist phytoplankton sample, a health food drink containing algae, pureed grass, and pureed leaves. Of these 5 samples, only the health food drink and pureed grass had any top matches with green algae from the spectral library, and none were matched with any of the cyanobacterial genera.

\subsection{Principle Component Cluster Analysis}

Cluster analysis showed clear differentiation of cyanobacterial samples from green algae using the first three 
principle components. For both the OMNIC (Figure 3) and OPUS (Figure 4) data sets, a tight clustering of cyanobacterial samples was seen, while green algae samples resulted in a clearly different, loose cluster. One sample initially labeled as green algae, however, was later found to be a species of protist phytoplankton rather than green algae. This sample was labeled as "other phytoplankton", and due to its closer relation to cyanobacteria, was not separated from the cyanobacterial cluster seen in the OMNIC data set, but was differentiated to a small degree in the OPUS data set.

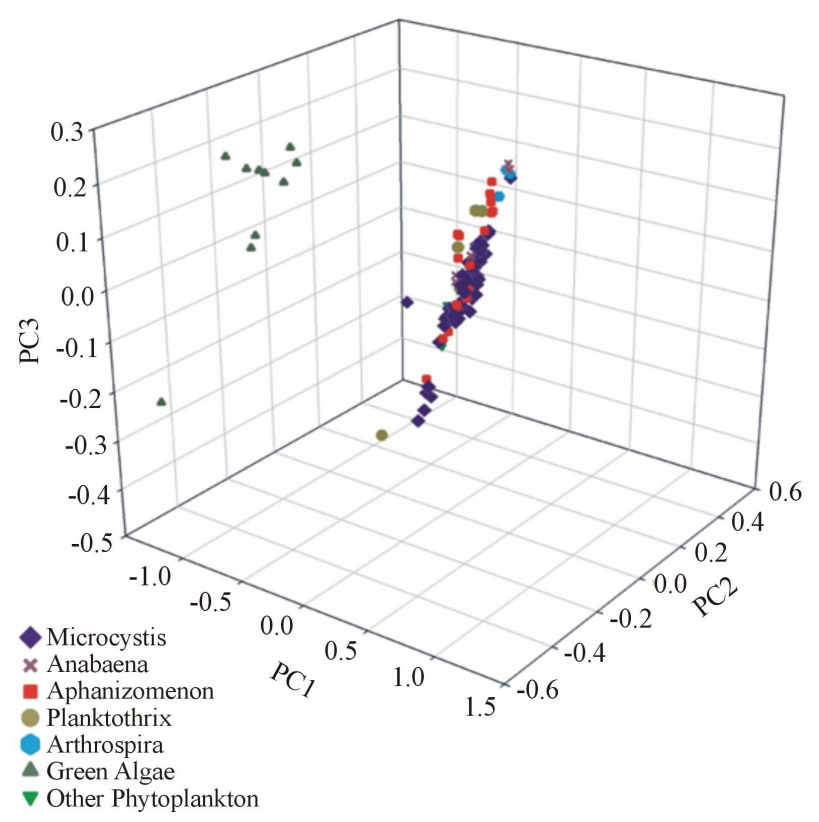

Figure 3. OMNIC 3D principle component cluster analysis.

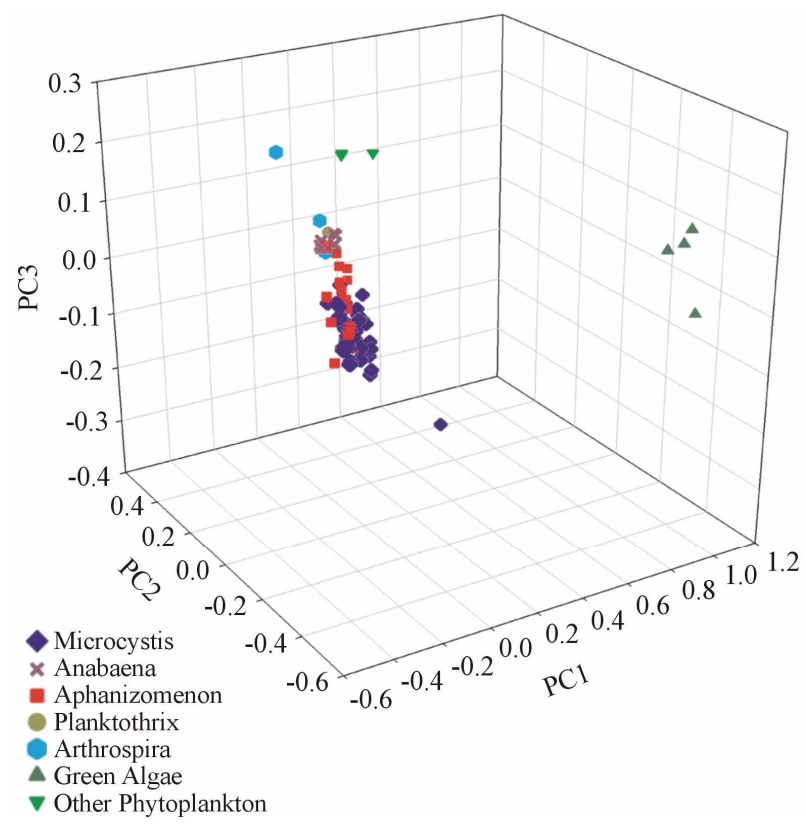

Figure 4. OPUS 3D principle component cluster analysis.

\subsection{Dendrograms}

Both the OMNIC and OPUS data sets resulted in dissimilarities between samples and genera that clearly distinguished cyanobacterial genera from green algae (Figures 5 and 6). Dendrograms created using average spectra for each genus expressed dissimilarities of small magnitudes between each cyanobacterial genera, and a much higher magnitude of difference between the cyanobacteria and green algae.

Dendrograms created on the whole data sets were consistent with those using the average spectra in regards to cyanobacterial and green algae differentiation. For both data sets, all green algae samples were grouped together and showed a large order of magnitude of difference between the cyanobacterial samples. The dissimilarities between the cyanobacteria and the "other phytoplankton" sample, however, were not enough to clearly differentiate it from the cyanobacterial samples in either data set.

Cophenetic correlation coefficients were recorded to verify that the dissimilarities expressed in the dendrograms are true representations of the original dissimilarities in the sample data. Dendrograms created from the average peak spectra resulted in high cophenetic correlation coefficients of 0.9890 and 0.9902 for the OMNIC and OPUS data sets, respectively. The values were slightly less for the whole sample set dendrograms, with the OMNIC data set cophenetic correlation coefficient being 0.9362 and the OPUS data set being 0.9383 .

\section{Discussion}

Due to the nature of environmental samples used in this study, sample quality was inevitably variable. To assess the practical application of FTIR default software algorithms in a diagnostic setting, sample exclusion criteria were not applied prior to screening, and all samples of variable quality were included. Therefore, some unsuccessful classifications were a result of poor sample quality, such as low cell density or samples containing sediments and other debris.

The reproduced results seen between the 12 samples that were re-scanned one year after their initial scan showed a remarkable degree of preservation of infrared spectral absorption characteristics once a sample has dried on filter paper.

The default software algorithms, principle component cluster analysis, and dendrograms were successful at differentiating between cyanobacterial samples and green algae, but were less successful at characterizing specific cyanobacterial genera. While Dean and Kanzis both report similar methods being successful at species differentiation, the methods as applied here to variable diagnostic samples were not successful at differentiating different types of cyanobacteria $[17,19]$. The method can therefore 

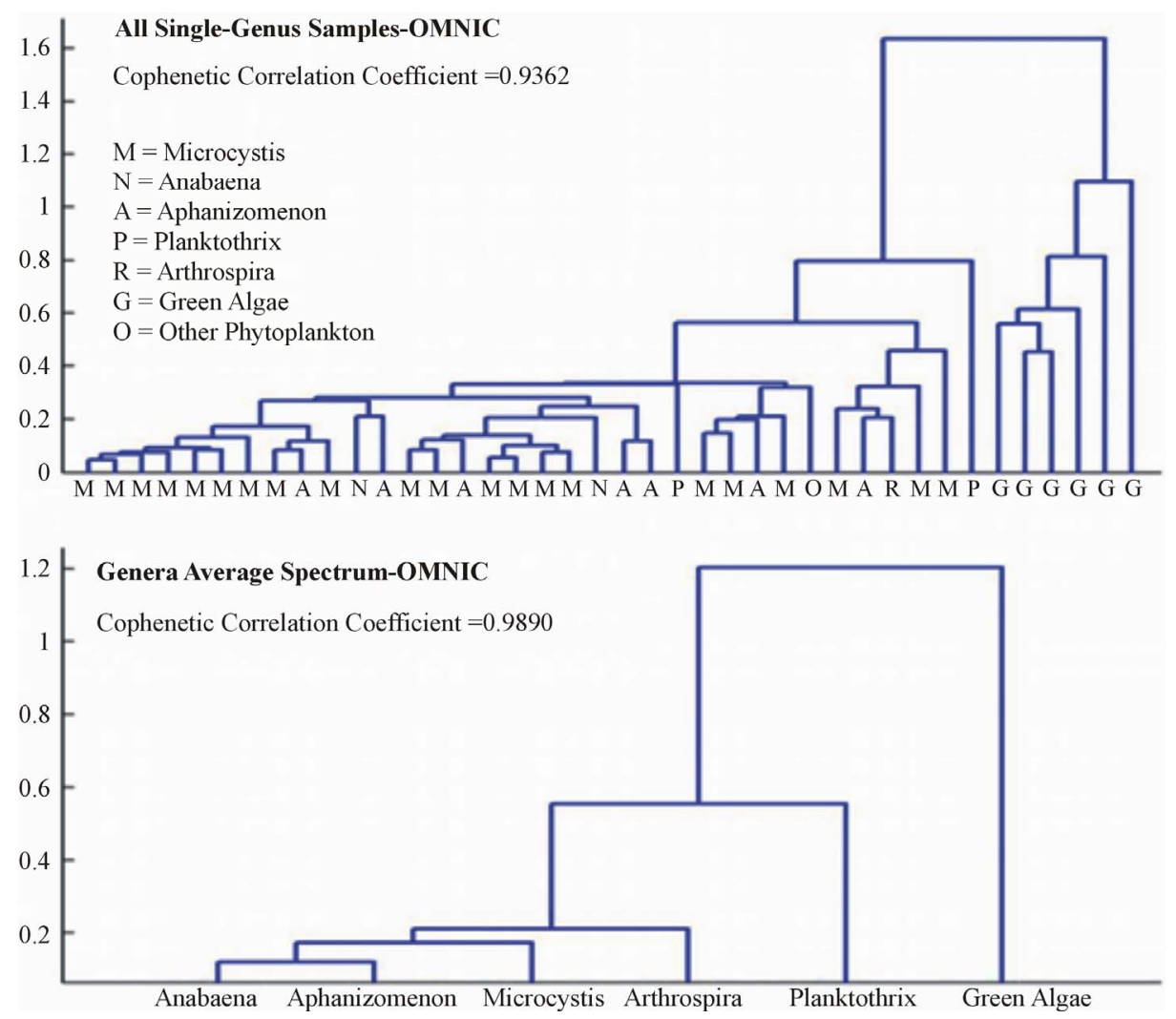

Figure 5. OMNIC dendrograms.
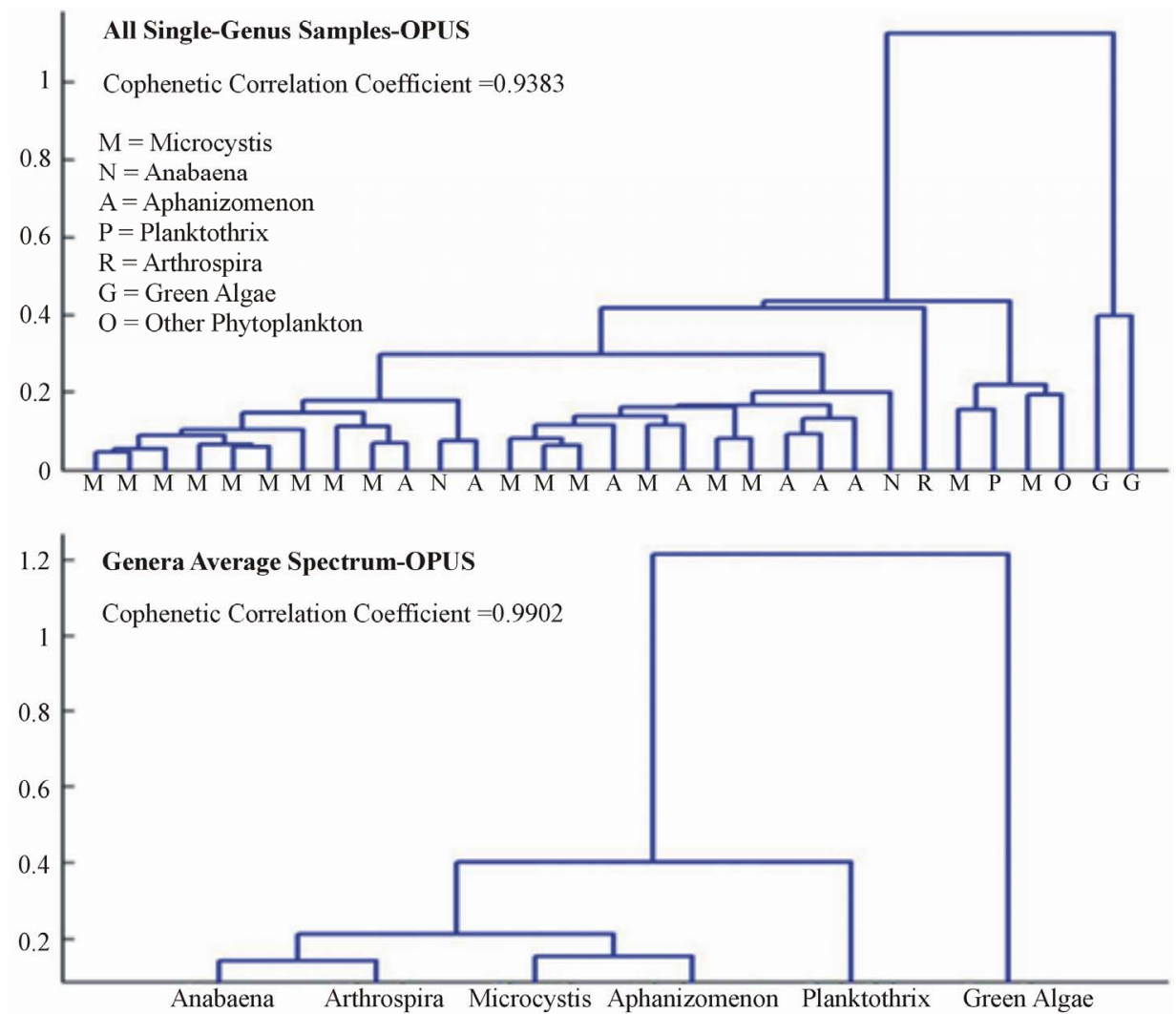

Figure 6. OPUS dendrograms. 
be applied as a practical screening method for the presence of cyanobacteria, but if taxonomic identification is needed, cyanobacteria must be classified using more specific methods such as microscopy.

\section{Conclusion}

Fourier-Transform Infrared Technology can be used in a relatively simple fashion to determine the presence or absence of potentially toxic cyanobacteria in environmental algal samples. Further manipulation of spectral data and strict sample quality control may be necessary to allow genus or species differentiation. While these methods cannot be used independently to fully characterize HABs, they do show the potential and practicality of FTIR as a screening tool.

\section{REFERENCES}

[1] O. A. Koksharova, "Application of Genetic Tools to Cyanobacterial Biotechnology and Ecology," In: P. M. Gault and H. J. Marler, Eds., Handbook on Cyanobacteria: Biochemistry, Biotechnology and Applications, Nova Science Publishers, New York, 2009, pp. 211-232.

[2] A. Gamboro, E. Barbaro, R. Zangrando and C. Barbante, "Simultaneous Quantification of Microcystins and Nodularin in Aerosol Samples Using High-Performance Liquid Chromatography/Negative Electrospray Ionization Tandem Mass Spectrometry," Rapid Communications in Mass Spectrometry, Vol. 26, No. 12, 2012, pp. 1497-1506. http://dx.doi.org/10.1002/rcm.6246

[3] G. A. Codd, L. F. Morrison and J. S. Metcalf, "Cyanobacterial Toxins: Risk Management for Health Protection," Toxicology and Applied Pharmacology, Vol. 203, No. 3, 2005, pp. 264-272. http://dx.doi.org/10.1016/j.taap.2004.02.016

[4] S. A. Oehrle, B. Southwell and J. Westrick, "Detection of Various Freshwater Cyanobacterial Toxins Using UltraPerformance Liquid Chromatography Tandem Mass Spectrometry," Toxicon, Vol. 55, No. 5, 2010, pp. 965-972. http://dx.doi.org/10.1016/j.toxicon.2009.10.001

[5] J. Al-Tebrineh, M. M. Gehringer, R. Ackaalan and B. A. Neilan, "A New Quantitative PCR Assay for the Detection of Hepatotoxigenic Cyanobacteria," Toxicon, Vol. 57, No. 4, 2011, pp. 546-554. http://dx.doi.org/10.1016/j.toxicon.2010.12.018

[6] A. Martins and V. Vasconcelos, "Use of qPCR for the Study of Hepatotoxic Cyanobacteria Population Dynamics," Archives of Microbiology, Vol. 193, No. 9, 2011, pp. 615-627. http://dx.doi.org/10.1007/s00203-011-0724-7

[7] J.-F. Briand, S. Jacquet, C. Bernard and J.-F. Humbert, "Health Hazards for Terrestrial Vertebrates from Toxic Cyanobacteria in Surface Water Ecosystems," Veterinary Research, Vol. 34, No. 4, 2003, pp. 361-377. http://dx.doi.org/10.1051/vetres:2003019

[8] D. van der Merwe, L. Sebbag, J. C. Nietfeld, M. T. Aubel, A. Foss and E. Carney, "Investigation of a Microcystisaeruginosa cyanobacterial Freshwater Harmful Algal
Bloom Associated with Acute Microcystin Toxicosis in a Dog," Journal of Veterinary Diagnostic Investigation, Vol. 24, No. 4, 2012, pp. 679-687. http://dx.doi.org/10.1177/1040638712445768

[9] B. M. Paddle, "Therapy and Prophylaxis of Inhaled Biological Toxins," Journal of Applied Toxicology, Vol. 23, No. 3, 2003, pp. 139-170. http://dx.doi.org/10.1002/jat.903

[10] S. W. Wright, D. P. Thomas, H. J. Marchant, H. W. Higgins, M. D. Mackey and D. J. Mackey, "Analysis of Phytoplankton of the Australian Sector of the Southern Ocean: Comparisons of Microscopy and Size Frequency Data with Interpretations of Pigment HPLC Data Using the 'CHEMTAX' Matrix Factorization Program," Marine Ecology Progress Series, Vol. 144, No. 1, 1996, pp. 285298. http://dx.doi.org/10.3354/meps 144285

[11] K. Rudi, F. Larsen and K. S. Jakobsen, "Detection of Toxin-Producing Cyanobacteria by Use of Paramagnetic Beads for Cell Concentration and DNA Purification," Applied and Environmental Microbiology, Vol. 64, No. 1, 1998, pp. 34-37.

[12] M. D. Mackey, D. J. Mackey, H. W. Higgins and S. W. Wright, "CHEMTAX - A Program for Estimating Class Abundances from Chemical Markers: Application to HPLC Measurements of Phytoplankton," Marine Ecology Progress Series, Vol. 144, No. 1, 1996, pp. 265-283. http://dx.doi.org/10.3354/meps144265

[13] J. An and W. W. Carmichael, "Use of a Colorimetric Protein Phosphatase Inhibition Assay and Enzyme Linked Immunosorbent Assay for the Study of Microcystins and Nodularins," Toxicon, Vol. 32, No. 12, 1994, pp. 14951507. http://dx.doi.org/10.1016/0041-0101(94)90308-5

[14] C. Sacksteder and B. A. Barry, "Fourier Transform Infrared Spectroscopy: A Molecular Approach to and Organismal Question," Journal of Phycology, Vol. 37, No. 2, 2001, pp. 197-199. http://dx.doi.org/10.1046/j.1529-8817.2001.037002197.x

[15] M. Giordano, M. Kansiz, P. Heraud, J. Beardall, B. Wood and D. McNaughton, "Fourier Transform Infrared Spectroscopy as a Novel Tool to Investigate Changes in Intracellular Macromolecular Pools in the Marine Microalga Chaetocerosmuellerii (Bacillariophyceae)," Journal of Phycology, Vol. 37, No. 2, 2001, pp. 271-279. http://dx.doi.org/10.1046/j.1529-8817.2001.037002271.x

[16] K. Stehfest, J. Toepel and C. Wilhelm, "The Application of Micro-FTIR Spectroscopy to Analyze Nutrient StressRelated Changes in Biomass Composition of Phytoplankton Algae," Plant Physiology and Biochemistry, Vol. 43, No. 7, 2005, pp. 717-726.

http://dx.doi.org/10.1016/j.plaphy.2005.07.001

[17] M. Kansiz, P. Heraud, B. Wood, F. Burden, J. Beardall and D. McNaughton, "Fourier Transform Infrared Microspectroscopy and Chemometrics as a Tool for the Discrimination of Cyanobacterial Strains," Phytochemistry, Vol. 52, No. 3, 1999, pp. 407-417. http://dx.doi.org/10.1016/S0031-9422(99)00212-5

[18] A. P. Dean, M. C. Martin and D. C. Sigee, "Resolution of Codimant Phytoplankton Species in a Eutrophic Lake Using Synchrotron-Based Fourier Transform Infrared 
Spectroscopy," Phycologia, Vol. 46, No. 2, 2007, pp. 151-159. http://dx.doi.org/10.2216/06-27.1

[19] A. P. Dean and D. C. Sigee, "Molecular Heterogeneity in Aphanizomenon Flos-aquae and Anabaena Flos-aquae (Cyanophyta): A Synchrotron-Based Fourier-Transform Infrared Study of Lake Micropopulations," European Journal of Phycology, Vol. 41, No. 2, 2006, pp. 201-212. http://dx.doi.org/10.1080/09670260600645907
[20] A. Parikh and D. Madamwar, "Partial Characterization of Extracellular Polysaccharides from Cyanobacteria," Bioresource Technology, Vol. 97, No. 15, 2006, pp. 18221827. http://dx.doi.org/10.1016/j.biortech.2005.09.008

[21] J. Coates, "Interpretation of Infrared Spectra, A Practical Approach," In: M. R. A., Ed., Encyclopedia of Analytical Chemistry, John Wiley \& Sons Ltd., Chichester, 2000, pp. 10815-10837. 